\title{
Renoprotective Effect of Plantago major Against Proteinuria and Apoptosis Induced by Adriamycin in Rat
}

\author{
Zohreh Naji Ebrahimi Yazd', Zahra Samadi Noshahr ${ }^{1,2 \dagger}$, Sara Hosseinian ${ }^{1,3}$, \\ Mohammad Naser Shafei ${ }^{1,4}$, Alireza Ebrahimzadeh Bideskan ${ }^{5}$, Reza Mohebbati', \\ Nazanin Entezari Heravi ${ }^{1}$, Samira Shahraki ${ }^{1,2}$, Somayeh Mahzari', \\ Abolfazl Khajavi Rad ${ }^{1,3 *}$
}

${ }^{1}$ Department of Physiology, faculty of Medicine, Mashhad University of Medical Sciences, Mashhad, Iran

${ }^{2}$ Student Research Committee, faculty of Medicine, Mashhad University of Medical Sciences, Mashhad, Iran

${ }^{3}$ Neurogenic Inflammation Research Center, Mashhad University of Medical Sciences, Mashhad, Iran

${ }^{4}$ Division of Neurocognitive Sciences, Psychiatry and Behavioral Sciences Research Center, Mashhad University of Medical Sciences,

Mashhad, Iran

${ }^{5}$ Department of Anatomy and Cell Biology, Faculty of Medicine, Mashhad University of Medical Sciences, Mashhad, Iran

\section{Key Words}

adriamycin, nephrotic syndrome, Plantago major, apoptosis, rat, extract

\begin{abstract}
Objective: Adriamycin (ADR) is an important anti-cancer drug which can cause renal toxicity. Given the known anti-inflammatory and antioxidant effects of Plantago major (P. major), the aim of this study was to determine the effects of hydroalcoholic extract of $P$. $m a$ jor on ADR- induced nephropathy in rats.

Methods: Fifty male Wistar albino rats were randomly divided into 5 groups including: control, ADR $(5 \mathrm{mg} / \mathrm{kg})$, ADR + P. major (600 and $1200 \mathrm{mg} / \mathrm{kg}$ ) and P. major ( 1200 $\mathrm{mg} / \mathrm{kg}$ ). The animals were treated with $P$. major extract for 5 consecutive weeks and ADR was intravenously injected on the 7th day of the study. Urine and serum samples were collected on days $0,14,21,28$, and 35 for
\end{abstract}

Received: Oct 17, 2018 Reviewed: Nov 06, 2018 Accepted: Feb 11, 2019

(c) This is an Open-Access article distributed under the terms of the Creative Common Attribution Non-Commercial License (http://creativecommons.org/licenses/by-nc/4.0/) which permits unrestricted noncommercial use, distribution, and reproduction in any medium, provided the original work is properly cited.

@ This paper meets the requirements of KS X ISO 9706, ISO 9706-1994 and ANSI/NISO Z39.48-1992 (Permanence of Paper). the measurement of serum cholesterol and albumin levels and urine protein excretion rate. At the end of the study, the left kidneys were removed for apoptosis assessment.

Results: Administration of ADR significantly decreased serum albumin level and increased serum cholesterol and urine protein excretion rate as well as, apoptotic cell numbers compared to the control group $(P<0.001)$ while had no effect on glomerular filtration rate $(P>0.05)$. Treatment with $P$. major , in both 600 and $1200 \mathrm{mg} / \mathrm{kg}$ doses, increased serum albumin level and decreased serum cholesterol concentration, urine protein excretion rate and as well as the number of apoptotic cell compared to the ADR group $(P<0.001)$.

Conclusion: Our results showed that the $P$. major extract effectively protects against ADR- induced nephropathy by reducing kidney apoptosis and improving renal functioning in rats.

\section{Introduction}

Nephrotic syndrome (NS) is a clinical term stating glomerular diseases characterized by intense protein-
*Corresponding Author

Abolfazl Khajavi Rad. Associate Professor of Physiology, Department of Physiology, Faculty of Medicine, Mashhad University of Medical Sciences Mashhad, Iran. Tel: +98-513-882-8565 Fax: +98-513-882-8564

E-mail:khajavirada@mums.ac.ir

$\dagger$ The author had an equal contribution as the first author

(c) 2019 Korean Pharmacopuncture Institute 
uria and consequent hypoproteinemia, edema and dyslipoproteinemia. It has been demonstrated that proteinuria is caused by glomerulosclerosis and loss of glomerular podocytes due to cell death/apoptosis [1]. Results from previous studies have demonstrated that oxidative stress, inflammation, and apoptosis are involved in the development of NS pathological features [2]. In the patients without effective treatment, NS leads to end-stage renal disease (ESRD) and require renal transplantation [1]. Despite the advances in NS studies in recent decades, the pathophysiology of this disease remains unknown and more studies are needed to clarify its exact pathophysiological mechanisms. Adriamycin (ADR) is a common anticancer drug that has been used for the treatment of a wide range of human solid tumors since 1969 [2]. However, despite having high antitumor efficacy, its effectiveness has been limited, because of renal, myocardia, testicular, and hematological toxicities [3]. However, in the animal studies ADR is used for induction of renal damage $[4,5]$ as well as hepatotoxicity [6] and cardiotoxicity. The cytotoxic effect of ADR is mediated via binding to DNA-associated enzymes (topoisomerase II) and inhibition of that, which could cause breakages of double strand in a DNA double helix and DNA intercalation, and initiation of intrinsic pathway of apoptosis. It has been suggested that the nephrotoxic effect of ADR is may be due to the formation of a large amount of toxic reactive oxygen species (ROS), which induces membrane lipid peroxidation and tissue injury $[7,8]$. The overproduction of ROS and imbalance between free oxygen radicals and antioxidants could trigger the immune response and mediate inflammation. Inflammatory mediators and cytokines play an important role in ADR-induced renal injury including increase glomerular capillary permeability as well as tubular atrophy and apoptosis $[2,9]$. The use of antioxidant compounds can be beneficial for nephrotoxicity caused by ADR. Plantago major ( $P$. major) is a member of the Plantaginaceae family; this plant is one of the great medicinal and commercial importance [10]. P. major has been used as an astringent, anti-inflammatory, antiviral, anaesthetic, antitumor, analeptic and anti-ulcer factor in traditional medicine [11]. This herbaceous plant contains caffeic mucilage, salicylic acid, pectin, saponin, terpenoids and flavonoids. In many studies, immunomodulatory, antimicrobial, antiulcer, anticancer, analgesic and antioxidant properties of $P$. major have been demonstrated [12-14].

The aim of this study was to investigate the possible protective effects of $P$. major on ADR- induced nephrotoxicity in rats.

\section{Materials and Methods}

\subsection{Extract Preparation}

P. major whole plant was collected from the nature around Mashhad and was identified by a botanist from the Herbarium of Ferdowsi University of Mashhad (Mashhad, Iran). For the preparation of the hydroalcoholic extract, $P$. major whole plant was separated, washed, shade-dried at room temperature, and then powdered. The powder was extracted in a Soxhlet extractor with ethanol (70\% v/v). After the extraction, the solution was purified using a rotary vacuum evaporator and kept at $4^{\circ} \mathrm{C}$ until use.

\subsection{Chemicals}

ADR was purchased from the Ebewe Pharma Company (Austria). TUNEL kit was obtained from Roche Company (In Situ Cell Death Detection Kit, Fluorescein, Roche Diagnostics, Mannheim, Germany). P. major whole plant was obtained from Medicinal Plants Division of Imam Reza Pharmacy.

\subsection{Animals}

Fifty male Wistar albino rats weighing $250 \pm 20$ g were obtained from Central Animal House of Faculty of Medicine, Mashhad University of Medical Sciences. The animals were maintained under standard condition (12-h light/ dark cycles at the temperature of $20-24^{\circ} \mathrm{C}$ ) with free access to food and water. All the experiments were approved by Ethical Committee of Mashhad University of Medical Sciences.

\subsection{Experimental Protocol}

In the present study, the animals were randomly divided into 5 groups $(\mathrm{n}=10)$ as follows:

Group A, control: received vehicle (tap water) for 5 consecutive weeks and saline was injected intravenously (i.v.) on the $7^{\text {th }}$ day of the experiment.

Group B, ADR: received vehicle (tap water) for 5 consecutive weeks and ADR was injected ( $5 \mathrm{mg} / \mathrm{kg}$, i.v) [15] on the $7^{\text {th }}$ day of the experiment.

Group C, $\boldsymbol{P}$. major 600 + ADR: received $P$. major extract (600 $\mathrm{mg} / \mathrm{kg}$, in drinking water) [5] for 5 consecutive weeks and i.v. injection of ADR on the $7^{\text {th }}$ day of the experiment.

Group D, $P$. major 1200+ADR: received $P$. major extract (1200 $\mathrm{mg} / \mathrm{kg}$, in drinking water) [5] for 5 consecutive weeks and i.v. injection of ADR on the $7^{\text {th }}$ day of the experiment.

Group E, $\boldsymbol{P}$. major 1200: received $P$. major extract (1200 $\mathrm{mg} / \mathrm{kg}$, in drinking water) and i.v. injection of saline on the $7^{\text {th }}$ day of the experiment.

Urine samples using individual metabolic cages and blood samples from the orbital sinus were collected at the begging of experiment (day 0) and days 14, 21, 28 and 35. (One, two, three and four weeks after ADR injection, respectively). Serum was separated by centrifugation at $3000 \times \mathrm{g}$ for $15 \mathrm{~min}$ and was stored at $-20^{\circ} \mathrm{C}$ until analysis.

At the end of the experiment, all the animals were anaesthetized and the left kidneys were immediately removed and fixed in $10 \%$ formalin for apoptosis assessment. Then, all the animals were humanly killed.

Serum was analyzed for albumin and total cholesterol concentration by a Convergys ${ }^{\bullet} 100$ Biochemistry Analyser using commercial kits (Pars Azmoon Company, Tehran, Iran). Urinary protein concentration was determined using Trichloroacetic acid precipitation assay [16]. 


\subsection{Detection of apoptotic nuclei in situ by TUNEL assay}

Left kidneys were fixed in $10 \%$ formalin, dehydrated in graded alcohols and embedded in paraffin, and then $5 \mu \mathrm{m}$ sections were prepared. The tissues were deparaffinized and rehydrated, followed by the incubation of the sections with proteinase $\mathrm{K}$ and immersion in methanol containing $3 \% \mathrm{H}_{2} \mathrm{O}_{2}$ for the inactivation of endogenous peroxidase. DNA fragmentation was detected using a commercial kit according to the manufacturer's instruction. A semi-quantitative analysis was performed by counting TUNEL-positive cells per unit area $\left(\mathrm{mm}^{2}\right)$ in the kidney at $\times 400$ magnification per field [17].

\subsection{Statistical analysis}

Data were analyzed by SPSS 16 . All the data were expressed as mean \pm SEM. Data related to the serum cholesterol, serum albumin, and urine protein excretion were analyzed by two-way mixed design ANOVA with time as repeated measure. Data on apoptosis were analyzed by one- way ANOVA. For further analyses, Tukey's post-hoc test was used when appropriate. $\mathrm{P}<0.05$ was considered as significant difference.

\section{Results}

\subsection{Serum cholesterol}

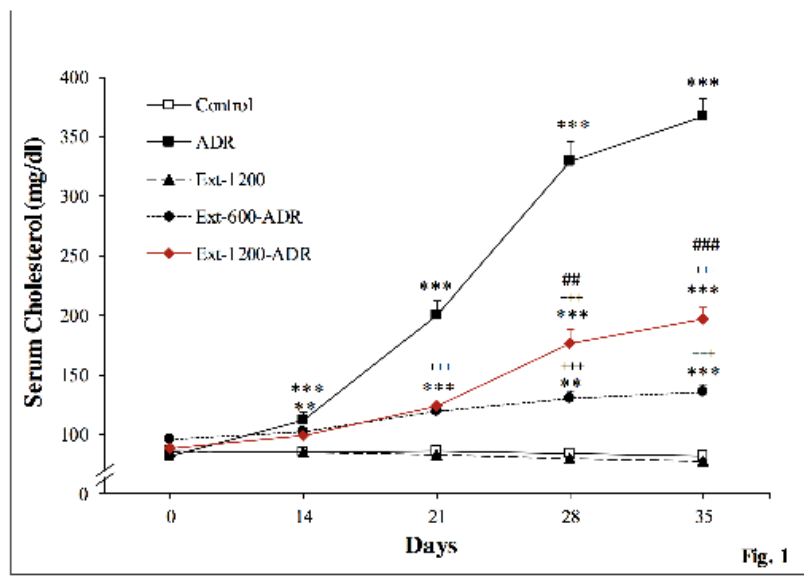

Figure 1 Change in serum cholesterol levels in the experimental groups. The data are shown as mean \pm SEM; $\mathrm{n}=10$. ${ }^{* *} P<0.01{ }^{* * *} P$ $<0.001$ vs. control group and ${ }^{+++} P<0.001$ vs. ADR group and ${ }^{\# \# \#+} P$ $<0.001$ vs. Ext-600-ADR group at each given time point. (Main effect by two-way mixed design ANOVA: treatment: $P<0.001$; time: $P<0.001$; interactions: $P<0.001)$.

Fig. 1 shows change in serum cholesterol levels during the experiment. A two-way mixed design ANOVA analyses revealed the significant main effect of time $(P<0.001)$ and treatment $(P<0.001)$ on the serum cholesterol levels. Also, there was a significant treatment $\times$ time interaction $(P<0.001)$. Tukey's post-hoc test revealed that serum cholesterol levels significantly increased in the ADR group compared to the control group on days 14 to $35(P<0.001$ for all the time points). Serum cholesterol levels signif- icantly reduced in the Ext-600-ADR and Ext- 1200-ADR as compared to the ADR group observed on days 21 to $35(P<0.001$ for all the time points); however, cholesterol level cannot reach the control level. Also the reduction of serum cholesterol level was significantly higher than the Ext-1200-ADR group in days 28 and 35 of experiment $(P$ $<0.001)$.

\subsection{Serum albumin}

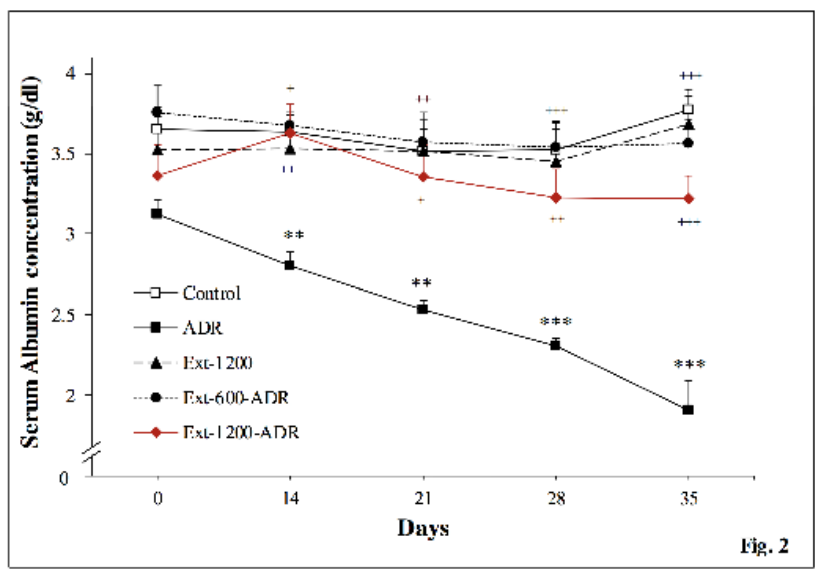

Figure 2 Serum albumin concentration in the experimental groups. The data are shown as mean \pm SEM; $\mathrm{n}=10$. ${ }^{* *} P<0.01$ ${ }^{* * *} P<0.001$ vs. control group and ${ }^{+} P<0.05,{ }^{++} P<0.01{ }^{+++} P<0.001$ vs. ADR group at each given time point. (Main effect by two-way mixed design ANOVA: treatment: $P<0.001$; time: $P<0.001$; interactions: $P<0.001)$.

As shown in Fig. 2, serum albumin concentration decreased over time in the ADR group. Two-way mixed ANOVA analyses revealed a significant time $(P<0.001)$, treatment $(P<0.001)$, and time $\times$ treatment interaction $(P<0.001)$. Subsequent analyses revealed that ADR caused a gradual decrease in the serum albumin concentration (days 14 and $21(P<0.01)$ and days 28 and $35(P<$ $0.001)$. Treatment of ADR rats with both 600 and $1200 \mathrm{mg} /$ $\mathrm{kg} P$. magor extract prevented, in a significant manner, from serum albumin decrease during the experiment.

\subsection{Urine protein excretion}

Fig. 3 shows the urine protein excretion rate in the rats measured on days 0, 14, 21, 28 and 35 after ADR injection. Analyses of urine protein excretion by two-way mixed designed ANOVA showed interactions between treatment and time $(P<0.001)$ and main effects of treatment $(P<$ $0.001)$ and time $(P<0.001)$. Additional analyses revealed that ADR significantly increased protein excretion on days $14(P<0.05), 21(P<0.001), 28(P<0.001)$ and 35 $(P<0.001)$ after ADR injection as compared to the control group. One-way ANOVA analyses at specific time points with Tukey's post-hoc test revealed that treatment with the extract of P. magor at the doses of 600 and $1200 \mathrm{mg} /$ $\mathrm{kg}$ decreased urine protein excretion. As compared to the ADR group, Ext-600-ADR group had less urine protein excretion on days $21(P<0.05), 28(P<0.001)$, and $35(P<$ $0.001)$. Also, urine protein excretion rate in the Ext-1200ADR group on days $28(P<0.05)$ and $35(P<0.001)$ was 
significantly lower than the one in the ADR group.

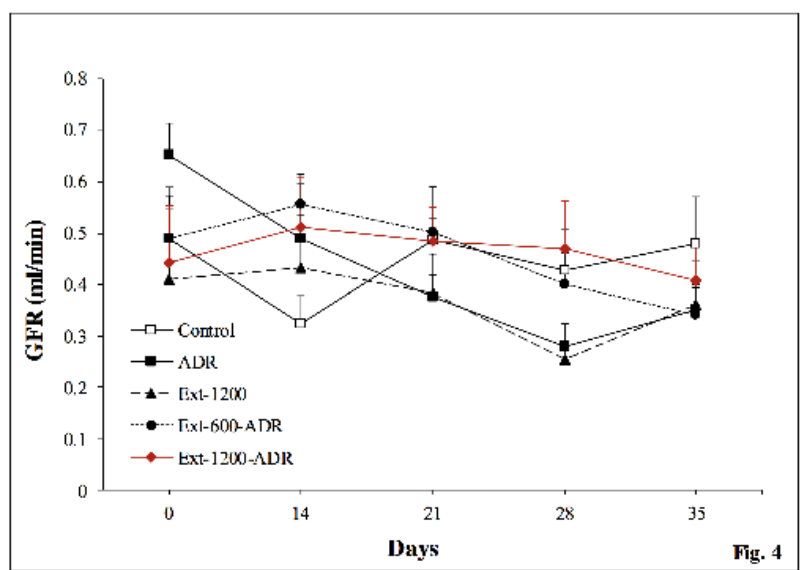

Figure 3 Change in proteinuria in experimental groups. The data are shown as mean \pm SEM; $\mathrm{n}=10 .{ }^{*} P<0.05$, ${ }^{* *} P<0.01$, ${ }^{* * *} P<0.001$ vs. control group and ${ }^{+} P<0.05,{ }^{++} P<0.001$ vs. ADR group at each given time point. (Main effect by two-way mixed design ANOVA: treatment: $P<0.001$; time: $P<0.001$; interactions: $P<0.001$ ).

\subsection{Glomerular filtration rate}

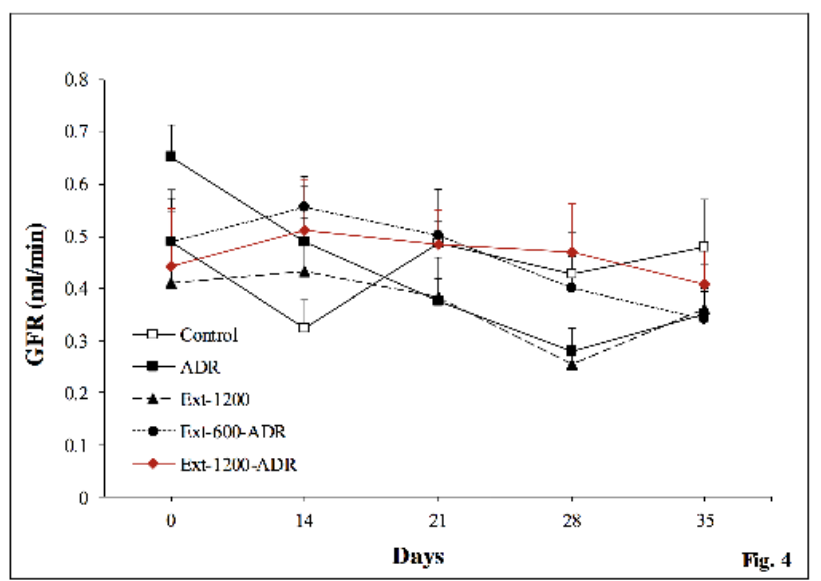

Figure 4 Glomerular filtration rate. The data are shown as mean \pm SEM; $\mathrm{n}=10$. (Main effect by two-way mixed design ANOVA: treatment: $P=0.688$; time: $P<0.05$; interactions: $P=0.249$ ).

Glomerular filtration rate (GFR) results are presented in Fig. 4. Analyses of GFR by two-way mixed designed ANOVA showed significant main effects of time $(P<0.05)$ but not treatment $(P>0.05)$ and time $\times$ treatment interactions $(P>0.05)$. Repeated measure ANOVA analysis also revealed that GFR in the ADR group significantly reduced in 28 and 35 days of experiment as compared to the day $0(P$ $<0.01$ and $P<0.05$ respectively).

\subsection{Number of renal apoptotic cells}

In ADR group, the number of TUNEL-positive cells was significantly higher than that of control group $(P<0.01)$. However, the number of renal apoptotic cells in ADR $+P$. major (600 and $1200 \mathrm{mg} / \mathrm{kg}$ ) groups was significantly decreased compared to ADR group $(P<0.001)$ (Fig. 5).

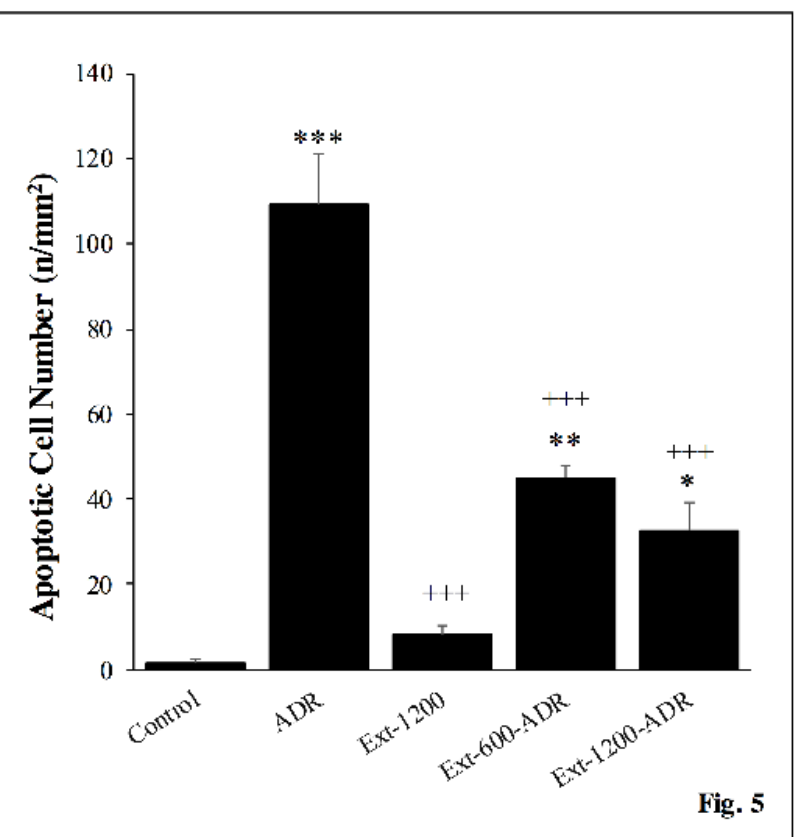

Figure 5 Comparison of TUNEL positive (apoptotic) cells per unit area ( $\mathrm{mm} 2)$ in the rat kidney of the all experimental groups. Values are expressed as mean \pm SEM. ${ }^{*} P<0.05,{ }^{* *} P<0.01,{ }^{* * *} P<0.001$ vs. control group and ${ }^{++} P<0.001$ vs. ADR group.

\section{Discussion}

In the present study a single dose injection of ADR significantly induced massive proteinuria significant elevation in serum cholesterol level and a significant reduction in serum albumin concentration when compared to the control animals. In our study, in a time-dependent manner, the oral administration of $P$. major extract significantly improved proteinuria and serum alterations in albumin and cholesterol levels, as well as the number of renal apoptotic cells in ADR treated rats. Although $P$. major extract in the both doses had beneficial effects on preventing of renal damage, however, the effects were varied in different parameters. For example, $1200 \mathrm{mg} / \mathrm{kg}$ dose prevent apoptosis better than $600 \mathrm{mg} / \mathrm{kg}$ dose, while $600 \mathrm{mg} / \mathrm{kg}$ dose was more effective in reducing of serum cholesterol levels. ADR-induced massive proteinuria compared to untreated animals, confirmed the accuracy of the present study in the induction of proteinuria. ADR administration was also associated with a significant elevation in serum cholesterol level and a significant reduction in serum albumin concentration when compared to the control animals. These functional abnormalities were accompanied by a marked increase in renal apoptotic cells. ADR-induced nephropathy is considered as a good experimental model for human minimal change disease characterized by albuminuria, proteinuria, hypoalbuminaemia, hyperlipidaemia, and edema [18]. It has been reported that ADR downregulates the expression of specific proteoglycans and some enzymes involved in glycosaminoglycans production. The glomerular charge selectivity was consequently eliminated, which caused flattening of podocyte foot processes and proteinuria. Based on our results, treatment of ADR-in- 
jected rats with $P$. major extract significantly improved the urinary excretion of protein, consistent with hypoalbuminemia. It seems that $P$. major extract could counteract the abnormal filtration of high molecular weight proteins from the glomerular filtration barrier. Furthermore, possibly due to its antioxidant and anti-inflammatory effects, $P$. major extract could suppress the destruction of glomerular basement membrane mediated by free radicals [5]. The average daily water intake was approximately 23 to $28 \mathrm{ml}$ in this study per rat. Drinking water at a concentration of 25 and $50 \mathrm{mg} / \mathrm{ml} P$. major extract, produce a dose of 600 and $1200 \mathrm{mg} / \mathrm{kg}$. In this concentration, P. major extract successfully dissolved in drinking water without any sedimentation or interference in water intake. In the same doses parhizgar et al. observed beneficial effects of $P$. major in Cisplatin induced nephrotoxicity, however, cisplatin causes acute renal failure (tubular damage) while in the present study ADR caused proteinuria (glomerular damage) [14]. In the same context, previous studies have reported the beneficial action of natural antioxidants against abnormal urinary protein excretion in ADR proteinuric experimental models $[5,19,20]$. In the present
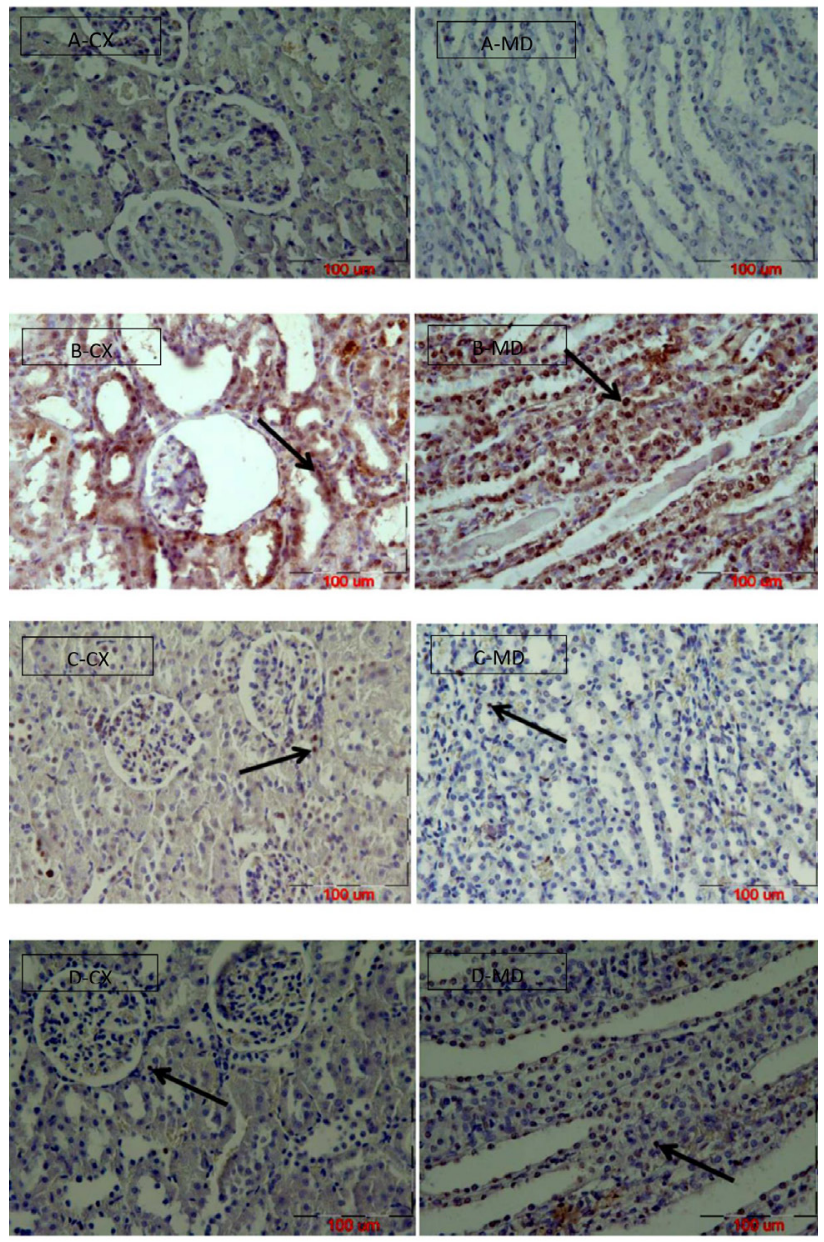

Figure 6 Photomicrographs showing the TUNEL positive cells in the renal cortex (CX) and medulla (MD) in different group of animals. Control group (A) showing normal morphology. ADR group (B). P. major extract (600, $1200 \mathrm{mg} / \mathrm{kg})$ (C \& D respectively) Arrows show apoptotic cell (magnification $400 \times$, scale bar $=100$ $\mu \mathrm{m})$. work, the renoprotective action of $P$. major extract was also observed by the time- dependent restoration of serum cholesterol in ADR injected rats. Recently, the hypolipidaemic properties of Plantago ovata husk and Plantago asiatica in diabetic rabbits and obese mice were reported [21, 22]. The exact mechanism for this hypolipidemic action of $P$. major extract in our study is not clear, but it might be contributed to its protection against proteinuria and subsequent hyperalbuminemia, which could minimize the compensatory production of cholesterol by the liver. However, other mechanisms including suppressing fatty acid synthesis, accelerating fatty acid $\beta$-oxidation, and stimulating cholesterol catabolism mainly by phenylpropanoid glycosides might be involved [22, 23].

Furthermore, the current work showed a significant decrease in the number of renal apoptotic cells of $P$. $m a$ jor-treated rats when compared to ADR group. Our results were consistent with those of Kho et al. (2017) who reported the anti-apoptotic effect of Plantago asiatica $L$. in puromycin aminonucleoside-induced nephrotic syndrome in rats [24]. Alleviation of renal cell apoptosis by $P$. major extract in ADR-treated rats may be attributed to its antioxidant and anti- inflammatory effects.

\section{Conclusion}

In conclusion, our results indicate that $P$. major extract markedly improved the ADR-induced nephropathy by reducing apoptosis and improving kidney functioning. This protective effect of $P$. major on ADR-induced nephropathy may be attributed to the antioxidant components of this plant. However, more studies are needed to clarify the exact mechanisms of beneficial actions of $P$. major in nephropathy associated with ADR.

\section{Acknowledgment}

This work was supported by the Research Council of Mashhad University of Medical Sciences. The results obtained in the present study are part of a MSc student's thesis (ID no. 931731).

\section{Conflict of interest}

The authir declare that there are no conflicts of interest. 


\section{References}

1. Kaneko Y, Narita I. Nephritis and nephrotic syndrome. Jpn J Nephrol. 2013;55(1):35-41.

2. El-Sayed ESM, Mansour AM, El-Sawy WS. Alpha lipoic acid prevents doxorubicin-induced nephrotoxicity by mitigation of oxidative stress, inflammation, and apoptosis in rats. J Biochem Mol Toxicol. 2017;31(9):e21940.

3. Ayla S, Seckin I, Tanriverdi G, Cengiz M, Eser M, Soner $B$, et al. Doxorubicin induced nephrotoxicity: protective effect of nicotinamide. Int J cell biol. 2011;2011:1-9.

4. Mohebbati R, Shafei MN, Beheshti F, Soukhtanloo M, Roshan NM, Anaeigoudari A, et al. Mixed hydroalcoholic extracts of Nigella sativa and Curcuma longa improves adriamycin-induced renal injury in rat. Saudi J Kidney Dis Transpl. 2017;28(6):1270.

5. Heravi NE, Hosseinian S, Yazd ZNE, Shafei MN, Bideskan AE, Shahraki S, et al. Doxorubicin- induced renal inflammation in rats: Protective role of Plantago major. Avicenna J phytomed. 2018;8(2):179.

6. Khazdair MR, Mohebbati R, Karimi S, Abbasnezhad A, Haghshenas M. The protective effects of Curcuma longa extract on oxidative stress markers in the liver induced by Adriamycin in rat. Physiol Pharmacol. 2016;20(1):31-7.

7. Lou H, Kaur K, Sharma AK, Singal PK. Adriamycin-induced oxidative stress, activation of MAP kinases and apoptosis in isolated cardiomyocytes. Pathophysiology. 2006;13(2):103-9.

8. Berthiaume JM, Wallace KB. Adriamycin-induced oxidative mitochondrial cardiotoxicity. Cell Biology and Toxicology. 2007;23(1):15-25.

9. Yilmaz S, Atessahin A, Sahna E, Karahan I, Ozer S. Protective effect of lycopene on adriamycin- induced cardiotoxicity and nephrotoxicity. Toxicology. 2006;218(2):164-71.

10. Haddadian K, Zahmatkash M. A review of Plantago plant. Indian J Trad Know. 2014;13(4):681-5.

11. Samuelsen AB. The traditional uses, chemical constituents and biological activities of Plantago major L. A review. J ethnopharmacol. 2000;71(1):1-21.

12. Gomez-Flores R, Calderon C, Scheibel L, Tamez $\$ Guerra $P$, Rodriguez-Padilla $C$, Tamez-Guerra $R$, et al. Immunoenhancing properties of Plantago major leaf extract. Phytother Res. 2000;14(8):617-22.

13. Núñez Guillén ME, da Silva Emim JA, Souccar C, Lapa AJ. Analgesic and Anti-inflammatory Activities of the Aqueous Extract of Plantago major L. Int J Pharmacog. 1997;35(2):99-104.

14. Parhizgar S, Hosseinian S, Hadjzadeh M-A-R, Soukhtanloo M, Ebrahimzadeh A, Mohebbati R, et al. Renoprotective Effect of Plantago Major Against Nephrotoxicity and Oxidative Stress Induced by Cisplatin. Iran J kidney dis. 2016;10(4).

15. Mohebbati R, Shafei MN, Soukhtanloo M, Roshan NM, Rad AK, Anaeigoudari A, et al. Adriamycin-induced oxidative stress is prevented by mixed hydro-alcoholic extract of Nigella sativa and Curcuma longa in rat kidney. Avicenna J phytomed. 2016;6(1):86.

16. Olson BJSC, Markwell J. Assays for Determination of
Protein Concentration. Current Protocols in Protein Science: John Wiley \& Sons, Inc.; 2001.

17. Abdel-Wahab A, Mahmoud W, Al-Harizy R. Comparative Renal Protective Effects of Canagliflozin and Telmisartan in a Rat Model of Diabetic Nephropathy. J Nephrol Renal Ther. 2016;2(10):2-8.

18. de Fátima Pereira W, Brito-Melo GEA, de Almeida CAS, Moreira LL, Cordeiro CW, Carvalho TGR, et al. The experimental model of nephrotic syndrome induced by Doxorubicin in rodents: an update. Inflammation Res. 2015;64(5):287-301.

19. Espinosa C, López-Jiménez JA, Pérez-Llamas F, Guardiola FA, Esteban MA, Arnao MB, et al. Long-term intake of white tea prevents oxidative damage caused by adriamycin in kidney of rats. J Sci Food Agric. 2016;96(9):3079-87.

20. You H, Lu Y, Gui D, Peng A, Chen J, Gu Y. Aqueous extract of Astragali Radix ameliorates proteinuria in adriamycin nephropathy rats through inhibition of oxidative stress and endothelial nitric oxide synthaseJ Ethnopharmacol. 2011;134(1):176-82.

21. Díez R, García JJ, Diez MJ, Sierra M, Sahagún AM, Calle ÁP, et al. Hypoglycemic and hypolipidemic potential of a high fiber diet in healthy versus diabetic rabbits. BioMed res int. 2013;2013:1-8.

22. Yang Q, Qi M, Tong R, Wang D, Ding L, Li Z, et al. Plantago asiatica L. Seed Extract Improves Lipid Accumulation and Hyperglycemia in High-Fat Diet-Induced Obese Mice. Int J mol sci. 2017;18(7):1393.

23. Egorov TA, Galkina T, Balashova T, Arsen'ev A, Nikonorova A, Babakov A, et al. Phenolic glycoside isolated from seeds of the greater plantain (Plantago major L.). Doklady Biochem Biophys. 2004;396(1-6):132-5.

24. Kho MC, Park JH, Han BH, Tan R, Yoon JJ, Kim HY, et al. Plantago asiatica L. Ameliorates Puromycin Aminonucleoside-Induced Nephrotic Syndrome by Suppressing Inflammation and Apoptosis. Nutr. 2017;9(4):386. 